\title{
Regional Lymph Nodes in Scalp Angiosarcomas: The Hidden Harbinger of Doom?
}

\author{
Miranda B. Lam, MD, MBA ${ }^{1,2}$, and Elizabeth H. Baldini, MD, MPH ${ }^{1,2}$ \\ ${ }^{1}$ Department of Radiation Oncology, Brigham and Women's Hospital/Dana Farber Cancer Institute, Boston, MA; ${ }^{2}$ Harvard \\ Medical School, Boston, MA
}

Angiosarcoma is a rare, malignant tumor of the vascular endothelium that accounts for $5 \%$ of cutaneous soft tissue sarcomas. About $60 \%$ of these cases occur in the head and neck area, most commonly located on the scalp and face. Three-year overall survival is poor and ranges from 10 to $50 \% .^{1-3}$ The optimal treatment strategy for this disease is not clear. Upfront surgical resection is a potential treatment approach, although several studies have shown a high local recurrence rate following wide excision and these recurrences are often associated with high morbidity. For this reason, as well as the fact that in many instances the disease may be too diffuse for upfront resection, systemic therapy is an attractive initial treatment modality followed by consolidative radiation therapy (RT) with or without surgery. Given the rarity of scalp angiosarcoma, prospective studies are lacking, but several retrospective studies indicate that patients who receive multimodality care appear to have lower local recurrence and improved survival. $^{1-5}$

The study by Kang et al. retrospectively analyzed 40 consecutive patients with scalp angiosarcoma at their institution. ${ }^{6}$ Interestingly, they found that regional lymph node (LN) metastases were seen in over $50 \%$ of patients with scalp angiosarcomas during their disease course, which is higher than previously reported. They also found that regional LNs were the most common form of initial metastases. Consistent with prior studies, overall survival

(C) Society of Surgical Oncology 2020

First Received: 7 March 2020;

Published Online: 6 April 2020

M. B. Lam, MD, MBA

e-mail: Miranda_Lam@dfci.harvard.edu was poor, with 2-year overall survival at $36 \%$. Distant metastases (DM) occurred in $70 \%$ of patients during their disease course.

The authors' institutional practice is to perform a surgical resection of the primary with neck dissection to remove involved LNs, as determined by fine needle aspiration (FNA) or highly suspicious LN on imaging. Surgery is followed by postoperative RT typically administered concurrently with taxane-based chemotherapy for patients they deem high risk. Of note, they treated $35 \%$ of patients in their series with surgery alone. Neck dissections were performed for either highly suspicious LN on imaging for which FNA was not required or suspicious $\mathrm{LN}$ determined to be positive by FNA. The extent of dissection depended on the primary site and could include ipsilateral parotidectomy (parotid and preauricular LNs), occipital and postauricular LNs, or ipsilateral neck LNs II-V. The authors found that patients who developed regional LN metastases all eventually developed distant metastatic disease within 3-6 months. In contrast, DM without regional $\mathrm{LN}$ involvement was only seen for $28 \%$ of patients. There was no difference in survival between patients with and without $\mathrm{LN}$ metastases in their series. The authors concluded that a reasonable approach to scalp angiosarcomas is initial curative surgery with prophylactic evaluation of regional LNs for pathological nodal staging and prognostication followed by adjuvant therapy with RT \pm chemotherapy.

While a number of prior single-institutional experiences on this topic have been published, one distinguishing aspect of this paper from prior studies is the high rate of LN metastases, which is reported to be over $50 \%$. In contrast, the incidence of $\mathrm{LN}$ involvement during the disease course in prior studies is highly variable, ranging from 5 to $35 \% .^{1-3}$ One possible cause for this variability is that most studies combined face and scalp angiosarcomas, which potentially 
carry different LN metastasis risk. Other possibilities are that studies represent a wide range of time intervals, patient populations, and treatment approaches. The observation made by authors regarding the high rate of LN metastases of scalp angiosarcomas raises two important questions. First, it suggests that patients may benefit from more comprehensive local treatment that includes local management of regional LNs, either with surgical neck dissection, RT, or both. Second, because the patients in this study with LN involvement universally developed metastatic disease, while the patients without $\mathrm{LN}$ involvement had a lower rate of DM development, the study raises the possibility that upfront assessment of LN involvement by imaging and FNA may provide useful prognostic information as well as identify patients who would benefit from upfront systemic treatment intensification or enrollment in a clinical trial.

Upfront clinical management of scalp angiosarcomas varies greatly among institutions. As mentioned above, neck staging was standard at the authors' institution for patients with suspicious LN on imaging and/or FNA diagnosis; however, many institutions follow a different strategy. For instance, our current institutional practice for treating patients with angiosarcoma of the face and scalp consists of neoadjuvant chemotherapy (typically taxanebased) followed by RT alone. Surgery is occasionally considered for smaller lesions (typically $<2 \mathrm{~cm}$ ). We do not routinely surgically stage the regional LNs. Historically, we more routinely utilized upfront surgical excision followed by RT to the postoperative bed (no LNs); however, given the high marginal recurrence rates following surgical excision, we have opted to utilize RT alone for local therapy after neoadjuvant chemotherapy. After a recent internal study of our scalp and face angiosarcoma patients, we found that among our 20 patients, 3 had LN involvement at diagnosis and 7 developed LN metastases during their treatment course. Because of this, we are now considering whether we should deliver consolidative whole-scalp RT and prophylactic coverage of regional LNs for most patients (except those with small unifocal disease). In light of the findings presented in this paper, it is possible that each institution will need to re-evaluate their institutional treatment patterns to determine how this new information fits into their current practice. Given the high rates of regional $\mathrm{LN}$ and metastatic disease development in cutaneous angiosarcoma of the scalp, it is clear that more effective systemic therapy is needed for this disease. Immunotherapy has shown some promise and may play a role. $^{7,8}$

In summary, this paper not only reinforces the need for better treatment for scalp angiosarcomas but also raises the possibility that LN involvement for scalp angiosarcoma may be more common than previously appreciated. This study raises the question of whether we should consider upfront surgical staging of regional neck LNs for prognostication, and potentially determining the need for local (comprehensive neck dissection or RT) or systemic treatment intensification or upfront enrollment of patients on clinical trials; however, the downside of such an approach is increased morbidity to patients from the surgical procedure. Additional data is needed to determine whether systematic assessment of regional LN involvement would allow for better stratification of patients to inform optimal treatment decisions. A randomized trial for scalp angiosarcomas is likely difficult due to low numbers and wide treatment variation across institutions. However, additional studies from other institutions to help inform the literature would be important to understand whether scalp angiosarcoma truly has such a high rate of LN involvement. Future studies should also consider breaking down populations with head and neck angiosarcoma to scalp versus face for more clarity on differences in treatment patterns and outcomes between the two groups.

DISCLOSURES Miranda B. Lam and Elizabeth H. Baldini declare no conflicts of interest.

\section{REFERENCES}

1. Guadagnolo BA, Zagars GK, Araujo D, Ravi V, Shellenberger TD, Sturgis EM. Outcomes after definitive treatment for cutaneous angiosarcoma of the face and scalp. Head Neck. 2011;33(5):661-7.

2. Patel SH, Hayden RE, Hinni ML, Wong WW, Foote RL, Milani S, et al. Angiosarcoma of the scalp and face. JAMA Otolaryngol Neck Surg. 2015;141(4):335.

3. Pawlik TM, Paulino AF, McGinn CJ, Baker LH, Cohen DS, Morris JS, et al. Cutaneous angiosarcoma of the scalp: a multidisciplinary approach. Cancer. 2003;98(8):1716-26.

4. Mark RJ, Tran LM, Sercarz J, Fu YS, Calcaterra TC, Juillard GF. Angiosarcoma of the head and neck. The UCLA experience 1955 through 1990. Arch Otolaryngol Head Neck Surg. 1993;119(9):973-8.

5. Jasper KD, Holloway CL, DeVries KJ, Truong PT. Local Relapse and Survival Outcomes in Patients with scalp sarcoma: a retrospective study of 95 patients treated in a provincial cancer care institution over 25 years. Cureus. 2019;11(7):e5236.

6. Kang Y, Bae J, Choi S, Jang K-T, Yu JI, Hong JY, et al. Regional lymph node metastasis of scalp angiosarcoma: a detailed clinical observation study of 40 cases. Ann Surg Oncol. 2020. https://doi. org/10.1245/s10434-020-08408-7.

7. Florou V, Rosenberg AE, Wieder E, Komanduri KV, Kolonias D, Uduman M, et al. Angiosarcoma patients treated with immune checkpoint inhibitors: a case series of seven patients from a single institution. J Immunother Cancer. 2019;7(1):213.

8. Vieira AC, Megid TBC, Melo R, Muniz D, Salgues ACR, Barbosa FG, et al. Response to anti-PD1 immunotherapy in patients with metastatic cutaneous sarcoma: case reports and literature review. Oxf Med Case Rep. 2020; 2020(1):omz138.

Publisher's Note Springer Nature remains neutral with regard to jurisdictional claims in published maps and institutional affiliations. 